\title{
Influence de la vitesse de dégradation ruminale des aliments composés sur le métabolisme énergétique de chèvres en début de lactation
}

\author{
S Giger-Reverdin, D Sauvant, S Raimbault
}

INRA, Laboratoire de Nutrition et Alimentation de I'INA-PG, 75231 Paris Cedex 05, France

L'impact de la vitesse de dégradation des aliments dans le rumen sur le métabolisme énergétique a été peu étudié jusqu'à présent.

Cet essai a été réalisé, en début de lactation, sur 2 lots de 7 chèvres multipares, équilibrés en fonction de leur potentiel laitier et de leur poids vif. Dès la mise-bas, les animaux ont reçu une ration complète composée d'ensilage de mais (54\%/MS), de paille de blé ( $6 \% / \mathrm{MS}$ ) et d'un aliment concentré. Le lot $R$ a reçu un mélange se dégradant a priori rapidement dans le rumen (blé, orge, avoine, pois, féverole) et le lot $L$, un mélange (maïs, sorgho, tourteau de soja, corn gluten meal) à dégradation théorique lente (Sauvant et al, 1985, Anim Feed Sci Technol, 13, 7-23). Les rations étaient iso-énergétiques $(0,97 \mathrm{UFL} / \mathrm{kg}$ $\mathrm{MS}$ ) et iso-protéiques ( $83 \mathrm{~g} \mathrm{PDI} / \mathrm{kg} \mathrm{MS}$ ).

Les données correspondent à des moyennes hebdomadaires individuelles des semaines 2 à 5 après la mise-bas. Au cours de la première et de la troisième semaines expérimentales, la composition en acides gras de la matière grasse du lait a été déterminée et certains constituants plasmatiques mesurés. Les analyses de variance ont été effectuées avec la procédure de mesures répétées du logiciel SAS.

Les quantités de matière sèche ingérée ( $R: 90,7$ vs $L: 91,0 \mathrm{~g} / \mathrm{kg} \mathrm{P}^{0,75}$ ) et donc celles d'énergie sont équivalentes entre les 2 lots. La production laitière du lot $R$ est inférieure à celle du lot $L\left(119\right.$ vs $\left.140 \mathrm{~g} / \mathrm{kg} P_{0,75}, P<0,08\right)$. Les teneurs du lait en lipides ( $R: 49,1 \mathrm{~g} / \mathrm{l}, \mathrm{L}: 50,1$ ), protéines ( $R: 32,2 \mathrm{~g} / \mathrm{l}, \mathrm{L}: 32,3)$ et lactose ( $\mathrm{R}: 50,1 \mathrm{~g} / \mathrm{l}, \mathrm{L}: 51,9$ ) ne diffèrent pas au seuil de $10 \%$. Cependant, la production de lactose du lot $\mathrm{R}$ est statistiquement inférieure à celle du lot $L(6,00$ vs $7,30 \mathrm{~g} / \mathrm{kg} P 0,75, P<0,05)$, ainsi que la production d'acides gras à 18 atomes de carbone $\left(1,60\right.$ vs $\left.2,18 \mathrm{~g} / \mathrm{kg} P^{0,75}, P<0,02\right)$. Les teneurs plasmatiques en bêta-hydroxybutyrate sont de $31,1(R)$ et $29,3(L) \mathrm{mg} / \mathrm{l}$ et en glucose de $0,591(R)$ et de $0,584(L) \mathrm{g} / \mathrm{l}$, et ne diffèrent pas statistiquement. Par contre, les chèvres du lot $R$ ont une teneur en acides gras non estérifiés (AGNE) significativement plus basse que celle du lot $L$ (188 vs $291 \mathrm{mmol} / \mathrm{l}$ ).

II ressort de cette étude que des amidons se dégradant lentement et incomplètement dans le rumen, comme le maïs et le sorgho, pourraient accroître le flux de glucose absorbé dans l'intestin grêle, ce glucose serait utilisé par l'animal pour synthétiser du lactose et ainsi produire du lait en plus grande quantité. Cet effet sur la production laitière favoriserait la mobilisation des réserves chez les animaux du lot $\mathrm{L}$ en début de lactation comme en témoignent les teneurs en AGNE et la production d'acides gras longs dans le lait, par rapport à des amidons à dégradation rapide, ce qui est en accord avec les données de Casper et al (1990, J Dairy Sci, 73, 10391050).

En conclusion, la vitesse de dégradation ruminale des aliments concentrés pourrait modifier le métabolisme énergétique et la composition du lait des ruminants en début de lactation. 